\title{
Estudo do Perfil Alimentar, Nutricional e da Qualidade de Vida de Frequentadores de uma Policlínica Universitária Localizada em São Paulo
}

\section{Study of the Food, Nutritional Profile and Quality of Life of Users of a University Polyclinics Located in São Paulo}

\author{
Denise Tatiana Mendes Vilagra ${ }^{1}$ \\ Gisleangela Santos d Lima ${ }^{1}$ \\ Thabata França da Silva ${ }^{1}$ \\ Danielle Pereira Martins ${ }^{2}$ \\ Marianne de Faria Chimello ${ }^{3}$ \\ Nyvian Alexandre Kutz ${ }^{4}$ \\ Leslie Andrews Portes 5 \\ Marcia Maria Hernandes de Abreu de Oliveira Salgueiro ${ }^{6}$
}

\section{RESUMO}

Objetivo: Avaliar o perfil de frequentadores com excesso de peso de uma policlínica universitária da zonal sul de São Paulo. Metodologia: Trata-se de um estudo transversal descritivo com coleta de dados antropométricos e a aplicação de questionário estruturado para as variáveis sociodemográficas, consumo alimentar e outros hábitos de vida e de qualidade de vida. Resultados: Ocorreu predominância do sexo feminino (81\%), classe socioeconômica C e D-E (72\%). O Índice de Massa Corporal médio foi $32,9 \mathrm{~kg} / \mathrm{m}^{2}$ e a prevalência de obesidade em mulheres e homens foi $74 \%$ e $64 \%$, respectivamente. $84 \%$ dos participantes necessitam fazer ajustes no consumo alimentar e em outros hábitos de vida. $64 \%$ referiram a qualidade de vida geral boa e muito boa. Conclusão: A maioria da amostra foi composta por mulheres, obesas, sem companheiro, com baixa escolaridade e de baixa classe socioeconômica, necessitando melhorar o consumo alimentar e outros hábitos de vida. No entanto, a qualidade de vida foi classificada como boa e muito boa.

\section{DESCRITORES}

Consumo alimentar. Qualidade de Vida. Estado Nutricional. Classe Social.

\begin{abstract}
Objective: To evaluate the profile of overweight users of a university polyclinic in the southern zone of São Paulo. Methodology: This is a descriptive cross-sectional study with anthropometric data collection and a structured questionnaire for sociodemographic variables, food consumption and other life habits and quality of life. Results: There was a predominance of females $(81 \%)$, socioeconomic class C and D-E $(72 \%)$. The average Body Mass Index was $32.9 \mathrm{~kg} / \mathrm{m}^{2}$ and the prevalence of obesity in women and men was $74 \%$ and $64 \%$, respectively. $84 \%$ of participants need to adjust the food intake and other lifestyle habits. $64 \%$ report good and very good overall quality of life. Conclusion: Most of the sample is composed of women, obese, without a partner, with low education and socioeconomic class. They need to improve food intake and other lifestyle habits and already have a good and very good quality of life.
\end{abstract}

\section{DESCRIPTORS}

Food Consumption. Quality of Life. Nutritional Status. Social Class.

\footnotetext{
${ }^{1}$ Nutricionista pelo Centro Universitário Adventista de São Paulo (UNASP).

${ }^{2}$ Graduanda em Nutrição pelo Centro Universitário Adventista. Aluna bolsista do Programa de Iniciação Científica do Curso de Nutrição do Centro Universitário Adventista de São Paulo, São Paulo-SP, Brasil.

${ }^{3}$ Graduanda em Nutrição pelo Centro Universitário Adventista. Aluna do Programa de Iniciação Científica do Curso de Nutrição do Centro Universitário Adventista de São Paulo, São Paulo-SP, Brasil.

${ }^{4}$ Nutricionista, Mestre em Nutrição Humana Aplicada pela Universidade de São Paulo. Docente do Curso de Nutrição do Centro Universitário Adventista de São Paulo, São Paulo-SP, Brasil.

${ }^{5}$ Educador Físico, Doutorando em Ciências da Saúde. Docente do Curso de Mestrado em Promoção da Saúde e do Curso de Nutrição do Centro Universitário Adventista de São Paulo, São Paulo-SP, Brasil.

${ }^{6}$ Nutricionista, Doutora e Mestre em Saúde Pública pela Universidade de São Paulo. Docente do Curso de Mestrado em Promoção da Saúde e do Curso de Nutrição do Centro Universitário Adventista de São Paulo, São Paulo-SP, Brasil.
} 
A população brasileira apresenta crescente excesso de peso e obesidade, segundo dados da Vigilância de Fatores de Risco e Proteção para Doenças Crônicas por Inquérito Telefônico, Vigitel Brasil $2018^{1}$. A pesquisa indica que mais da metade dos brasileiros $(55,7 \%)$ apresentam peso acima do recomendado e $19,8 \%$ estão obesos. No ano de $2006,42,6 \%$ dos brasileiros apresentavam sobrepeso e 11,8\% obesidade, um crescimento de $30,8 \%$ e $67,8 \%$, respectivamente em apenas doze anos ${ }^{1}$. Esses são dados preocupantes e seguem tendências mundiais, quer em países desenvolvidos ou em desenvolvimento ${ }^{2}$.

O sobrepeso e a obesidade podem ser definidos pelo excessivo acúmulo de tecido adiposo de modo a comprometer a saúde do indivíduo ${ }^{3}$ e são fatores de risco interligados a outras doenças crônicas não transmissíveis (DCNT), como a hipertensão, o diabetes, câncer e a osteoartrite, piorando a qualidade de vida e aumentando o risco de morte prematura. A obesidade ainda se destaca por ser, simultaneamente, doença e fator de risco para outras doenças desse grupo ${ }^{4}$. O diagnóstico é feito utilizando o parâmetro recomendado pela Organização Mundial da Saúde (OMS), o Indice de Massa Corporal (IMC) $)^{5}$.

Entre os principais fatores que contribuem para o desenvolvimento da epidemia da obesidade estão o elevado consumo de alimentos com grande densidade energética e o estilo de vida sedentário, tendo o maior índice em populações com maior grau de pobreza e menor nível educacional ${ }^{3}$. Pode-se explicar essa associação pela maior palatabilidade e pelo baixo custo de alimentos de grande densidade energética, conseguida em alimentos industrializados ${ }^{6}$.

É preciso conhecer o perfil desses indivíduos para que as ações de promoção, prevenção e adoção de políticas públicas para a promoção da saúde ${ }^{6}$. A educação em saúde é uma ferramenta importante para a construção de estratégias para mudanças no comportamento alimentar e estilo de vida ${ }^{7}$.

Com isso, o objetivo deste trabalho foi investigar o perfil alimentar, nutricional e de qualidade de vida de homens e mulheres que frequentam a policlínica universitária.

\section{METODOLOGIA}

Estudo transversal realizado com frequentadores de uma policlínica universitária da zona sul de São Paulo. Os critérios para elegibilidade incluíram indivíduos com idade igual ou superior a 18 anos, de ambos os sexos e com excesso de peso.

A pesquisa foi submetida tanto ao Comitê de Ética em Pesquisa (CEP) do Centro Universitário Adventista de São Paulo (UNASP), sob CAAE no 75449617.8.0000.5377, como também à coordenação da policlínica universitária, e somente teve início após a aprovação de ambos. A divulgação foi feita junto aos profissionais e recepcionistas da policlínica e com a fixação de cartazes contendo informações da pesquisa. Os interessados em participar, informaram nome e telefone de contato para as recepcionistas entregarem aos pesquisadores.

Através de contato telefônico com os interessados, foi agendado dia e horário com 95 frequentadores. Destes, 12 não compareceram e 19 recusaram participar da pesquisa, 
totalizando 64 entrevistados entre adultos e idosos. Entre estes, 3 questionários estavam incompletos pois os frequentadores alegaram não ter tempo para a finalização do mesmo e 4 não preenchiam os critérios de inclusão. $A$ Figura 1 apresenta o fluxograma do processo amostral.

As entrevistas ocorreram nos meses de outubro e novembro de 2017, caracterizando uma amostra por conveniência. A coleta de dados ocorreu em uma sala reservada, no prédio da policlínica, onde foram prestados todos os esclarecimentos sobre o estudo. Somente foram incluídos como participantes indivíduos que assinaram o Termo de Consentimento Livre e Esclarecido (TCLE) em duas vias iguais. $\mathrm{O}$ instrumento utilizado foi um questionário estruturado, que permitiu coletar informações referentes a identificação do indivíduo (nome completo, endereço e telefone para contato, idade, dados antropométricos, sexo), dados para a classificação socioeconô- mica, consumo alimentar e qualidade de vida.

Foram excluídos da amostra: gestantes, indivíduos não aptos à responder os questionários (devido a incapacidade de compreensão e comunicação verbal) e aqueles com limitações físicas que impediam a aferição dos dados antropométricos.

$\mathrm{Na}$ estimativa dos dados antropométricos, o peso corporal foi medido de acordo com Frisancho ${ }^{8}$, por meio de balança eletrônica da marca Filizola fixa com capacidade de 150 $\mathrm{kg}$ e sensibilidade de $100 \mathrm{~g}$. Os participantes foram orientados quanto a posição correta - posicionado no centro da balança com o peso distribuído entre ambos os pés, com roupas leves (sem casaco ou paletó) e sem calçados. A medida de estatura também foi realizada de acordo com Frisancho ${ }^{8}$, com estadiômetro fixo. Foram tomadas duas medidas sucessivas e calculada a média entre elas, desde que não houvesse diferença maior do que um centímetro.

Figura 1. Fluxograma do processo amostral de frequentadores de uma Policlínica Universitária da zona sul de São Paulo (SP), 2018.

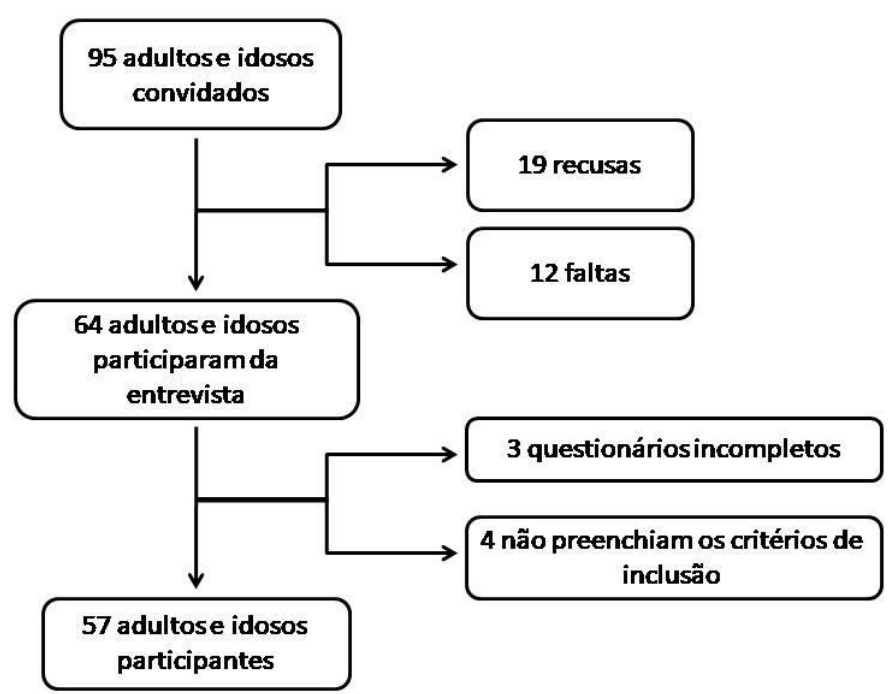


Para medir a circunferência da cintura (CC) foi utilizada a Norma Técnica do Sistema de Vigilância Alimentar e Nutricional - SISVAN $^{9}$, posicionado o indivíduo em pé, com os braços estendidos ao longo do corpo e pés paralelos, a roupa afastada de forma que a região da cintura fique despida. Marcado com caneta o ponto médio entre a extremidade da última costela e a crista ilíaca. Posicionando a fita métrica no ponto determinado, ao redor da cintura e realizada a leitura após uma expiração. A classificação, de acordo com as recomendações da OMS, considerando-se risco aumentado para doença metabólica, quando CC igual ou superior a $94 \mathrm{~cm}$ (homens) e $80 \mathrm{~cm}$ (mulheres); e risco aumentado substancialmente para CC igual ou superior a $102 \mathrm{~cm}$ em homens e $88 \mathrm{~cm}$ em mulheres ${ }^{10}$.

A determinação da classificação do Índice de Massa Corporal (IMC) foi feita seguindo as especificações da OMS para a população adulta (>19 anos), com classificação em baixo peso: IMC < 18,5 kg/m²; eutrofia: IMC 18,5 a 24,9 kg/m²; sobrepeso: IMC 25 a $29,9 \mathrm{~kg} / \mathrm{m}^{2}$; obesidade grau I: 30 a $34,9 \mathrm{~kg} / \mathrm{m}^{2}$; obesidade grau II: 35 a $39,9 \mathrm{~kg} / \mathrm{m} 2$ e obesidade grau III: $\geq 40 \mathrm{~kg} / \mathrm{m} 211$. Para a população idosa, empregou-se faixas definidas pela Organização Pan-Americana de Saúde (OPAS), onde baixo peso: IMC < 23; Peso adequado: IMC 23 a $28 \mathrm{~kg} / \mathrm{m}^{2}$; Excesso de peso: IMC $>28$ a 30 kg/m²; Obesidade: $I M C>30$ kg/m $/ \mathrm{m}^{2}$.

A classificação socioeconômica foi estabelecida através da coleta de informações sobre a escolaridade do chefe da família e os bens de consumo presentes na residência do usuário e acesso a serviços público, segundo o Critério de Classificação Econômica Brasil, adotado pela Associação Brasileira de Empresas de Pesquisa ${ }^{13}$.
Para avaliação do consumo alimentar foi utilizado o questionário adaptado, "Como está a sua alimentação", parte integrante de documento produzido pelo Ministério da Saúde do Brasil, "Guia alimentar: como ter uma alimentação saudável", que consiste em 18 perguntas do tipo fechadas, que recebem determinada pontuação conforme escolha da alternativa. Na somatória final da pontuação é possível aferir o resultado: Até 28 pontos "Atenção" - a alimentação deve sofrer alterações para tornar-se saudável; de 29 a 42 pontos "Precisa Melhorar" - alguns pontos precisam ser melhorados; e a partir de 43 pontos "Parabéns" - a alimentação é considerada saudável ${ }^{14}$.

$\mathrm{Na}$ avaliação da qualidade de vida, recorreu-se ao instrumento proposto pela OMS para adultos,o Word Health Organization Quality of Life Instrument Bref (WHOQOL-bref). O WHOQOL-bref é uma versão abreviada do questionário $W H O Q O L$, e que consiste de 26 questões sendo duas a respeito da qualidade de vida em geral e as demais representam cada uma das 24 facetas que compõem o instrumento original, divididas em quatro domínios: físico (dor física e desconforto, dependência de medicação/tratamento, energia e fadiga, mobilidade, sono e repouso, atividades da vida cotidiana, capacidade para o trabalho), psicológico (sentimentos positivos e negativos, espiritualidade/crenças pessoais, aprendizado/memória/concentração, aceitação da imagem corporal e aparência, autoestima), relações sociais (relações pessoais, atividade sexual, suporte/apoio social) e ambiente (segurança física, ambiente físico, recursos financeiros, novas informações/habilidades, recreação e lazer, ambiente no lar, cuidados de saúde, transporte) ${ }^{15}$. 
Todas as análises estatísticas foram feitas por meio dos pacotes estatísticos Graph Pad Prism, versão 6.0, e SPSS, versão 22.0, ambos para Windows. A normalidade dos dados foi testada pelo método de D'Agostino-Pearson. Os dados numéricos foram expressos como média \pm desvio-padrão (DP), e os dados categóricos como frequência, porcentagens e respectivos intervalos de confiança de 95\% (IC95\%). As comparações entre mulheres e homens foram realizadas por meio do teste $t$ de Student ou Mann-Whitney, conforme a necessidade. As variáveis categóricas foram analisadas por meio do teste do qui-quadrado. Adicionalmente, realizou-se correlações entre os testes buscando determinar possíveis associações. Os coeficientes de correlação ( $r$ ) foram interpretados segundo proposto por Cohen (1988, In: Batterham e Hopkins $)^{16}$ e adaptado por Mukaka ${ }^{17}: r<0,20$ muito fraca, $r<0,40$ fraca, $r<0,60$ moderada, $r<0,80$ forte e $\geq 0,80$ muito forte. Em todos os casos foram considerados estatisticamente diferentes quando $p \leq 0,05$. A percepção de qualidade de vida em cada um dos quatro domínios e do geral foram agrupados em 5 categorias: até 19,9 pontos: muito baixa, de 20,0 a 39,9 pontos: baixa, de 40,0 a 59,9 pontos: regular, de 60,0 a 79,9 pontos: boa e de 80,0 a 100,0 pontos: muito boa.

\section{RESULTADOS}

As principais características da população do estudo são apresentadas na Tabela 1. Com a avaliação de 57 indivíduos, a amostra revelou-se predominantemente feminina (81\%), com média de idade de 53,4 anos (DP= $11,4)$, variando de 18 a 68 anos. Um terço dos entrevistados é idoso. Mais da metade das pessoas ( $51 \%$ ) vive sem companheiro, o chefe da família apresenta menos de 12 anos de estudo $(56 \%)$ e pertencem às classes socioeconômicas C e D-E (72\%). A prevalência de obesidade foi de $74 \%$ nas mulheres e $64 \%$ nos homens. O IMC médio foi de $32,9 \mathrm{~kg} / \mathrm{m}^{2}$ $(\mathrm{DP}=4,4)$. Quanto a circunferência da cintura (CC), as mulheres também apresentaram maiores prevalências de risco aumentado substancialmente em relação aos homens, com $87 \%$ versus $55 \%$, com diferenças estatisticamente significativas $(p=0,04)$.

$\mathrm{Na}$ análise do consumo alimentar, $84 \%$ dos entrevistados foram classificados em "Atenção" e "Precisa melhorar", sendo mais acentuada nos homens, com $91 \%$ de inadequação. Os homens, na sua totalidade, mostraram inadequação no consumo diário de porções de frutas $(p=0,027)$ e leguminosas $(p=0,009)$. Também demonstraram maior consumo de salgadinhos, frituras e embutidos de no mínimo duas vezes por semana (64\%) contra $28 \%$ das mulheres $(p=0,027)$. Com relação a adição de sal em refeições já prontas, $18 \%$ dos homens tem essa prática em relação a $2 \%$ das mulheres $(p=0,033)$.

Em relação à escolaridade, não foram observadas diferenças significantes em relação ao IMC, CC, consumo alimentar e outros hábitos de vida e qualidade de vida.

Quanto à qualidade de vida, para os domínios físico,psicológico e das relações sociais, a maioria dos frequentadores foi classificada na categoria boa + muito boa ( $51 \%$ $70 \%$ e $68 \%$, respectivamente), enquanto para o domínio ambiente a distribuição foi entre as categorias regular $(49 \%)$ e boa + muito boa (47\%). Na classificação geral da qualidade 
Tabela 1. Características antropométricas, sociodemográficas e consumo alimentar de frequentadores de uma policlínica universitária da zona sul de São Paulo, 2018.

\begin{tabular}{lccc}
\hline \multicolumn{1}{c}{ Variáveis } & Todos & Mulheres & Homens \\
\hline \multicolumn{4}{c}{ Antropometria } \\
$\mathrm{n}(\%)$ & $57(100)$ & $46(81)$ & $11(19)$ \\
Idade & $53,4 \pm 11,4$ & $55 \pm 9,4$ & $46,6 \pm 14,9$ \\
Estatura & $160,1 \pm 9,1$ & $157,1 \pm 6,9$ & $172,6 \pm 6,3$ \\
Peso & $84,1 \pm 11,8$ & $82,2 \pm 11,4$ & $92,2 \pm 10,6$ \\
IMC & $32,9 \pm 4,4$ & $33,4 \pm 4,6$ & $30,9 \pm 2,9$ \\
& Classificação IMC & & \\
Sobrepeso & $\mathrm{n}(\%)$ & & $04(36)$ \\
Obesidade & $16(28)$ & $12(26)$ & $07(64)$
\end{tabular}

Circunferência da cintura

$\begin{array}{lccc} & n(\%) & \\ \text { Risco aumentado } & 09(16) & 05(10) & 04(36) \\ \text { Risco aum. subs. } & 46(81) & 40(87) & 06(55)^{*}\end{array}$

\begin{tabular}{lccc}
\multicolumn{4}{c}{ Classificação socioeconômica } \\
$\mathrm{n}(\%)$ & $12(26)$ & $04(36)$ \\
$\mathrm{A}+\mathrm{B}$ & $16(28)$ & $27(59)$ & $07(64)$ \\
C & $34(60)$ & $07(15)$ & - \\
D-E & $07(12)$ &
\end{tabular}

\begin{tabular}{llll} 
& \multicolumn{3}{c}{ Escolaridade do participante } \\
& \multicolumn{1}{c}{$\mathrm{n}(\%)$} & \\
$>8$ anos & $30(53)$ & $27(59)$ & $3(27)$ \\
$>8$ anos & $27(47)$ & $19(41)$ & $8(73)$
\end{tabular}

\begin{tabular}{llll}
\multicolumn{4}{c}{$\begin{array}{c}\text { Estado civil } \\
\mathrm{n}(\%)\end{array}$} \\
Sem companheiro & $28(51)$ & $24(52)$ & $04(36)$ \\
Com companheiro & $29(49)$ & $22(48)$ & $07(64)$ \\
& $\begin{array}{l}\text { Consumo alimentar } \\
\mathrm{n}(\%)\end{array}$ & \\
Atenção & $01(72)$ & $36(78)$ & $05(45,5)$ \\
Precisa melhorar & $07(12)$ & $02(4)$ & $05(45,5)$ \\
Parabéns & $09(16)$ & $08(18)$ & $01(9)$
\end{tabular}

* $p<0,05$. Risco aum. subs.: risco aumentado substancialmente 
Tabela 2. Questões sobre o consumo alimentar e hábitos de vida de frequentadores de uma policlínica universitária da zona sul de São Paulo, 2018.

\begin{tabular}{|c|c|c|c|c|c|c|}
\hline \multirow{3}{*}{ Questões } & \multirow{2}{*}{\multicolumn{2}{|c|}{$\begin{array}{c}\text { Todos } \\
\text { Adequado Inadequado }\end{array}$}} & \multicolumn{2}{|c|}{ Mulheres } & \multicolumn{2}{|c|}{ Homens } \\
\hline & & & Adequado & Inadequado & Adequado & Inadequado \\
\hline & $\mathrm{n} \quad(\%)$ & $\mathrm{n} \quad(\%)$ & $\mathrm{n} \quad(\%)$ & $\mathrm{n} \quad(\%)$ & $\mathrm{n} \quad(\%)$ & $\mathrm{n} \quad(\%)$ \\
\hline Q1. Consumo diário de frutas & $15(26)$ & $42(74)$ & $15(33)$ & $31(67)$ & - & $11(100)^{*}$ \\
\hline $\begin{array}{l}\text { Q2. Consumo diário de } \\
\text { legumes e verduras }\end{array}$ & $10(17)$ & $47(82)$ & $08(17)$ & $38(83)$ & $02(18)$ & $09(82)$ \\
\hline Q3. Consumo de leguminosas & $19(33)$ & $38(67)$ & $19(41)$ & $27(59)$ & - & $11(100)^{*}$ \\
\hline $\begin{array}{l}\text { Q4. Consumo de arroz, milho e } \\
\text { outros cereais }\end{array}$ & $24(42)$ & $33(58)$ & $18(39)$ & $28(61)$ & $06(55)$ & $05(45)$ \\
\hline Q5. Consumo diário de carnes & $20(35)$ & $37(65)$ & $16(35)$ & $30(65)$ & $04(36)$ & $07(64)$ \\
\hline $\begin{array}{l}\text { Q6. Retirada de gordura } \\
\text { aparente de carnes }\end{array}$ & $44(77)$ & $13(23)$ & $37(80)$ & $09(20)$ & $07(64)$ & $04(36)$ \\
\hline $\begin{array}{l}\text { Q7. Frequência de consumo de } \\
\text { peixes }\end{array}$ & $07(12)$ & $50(88)$ & $07(15)$ & $39(85)$ & - & $11(100)$ \\
\hline $\begin{array}{l}\text { Q8. Consumo diário de leite e } \\
\text { derivados }\end{array}$ & $04(7)$ & $53(93)$ & $04(9)$ & $42(91)$ & - & $11(100)$ \\
\hline Q9. Tipo de leite e derivados & $19(33)$ & $38(67)$ & $15(33)$ & $31(67)$ & $04(36)$ & $007(64)$ \\
\hline $\begin{array}{l}\text { Q10. Frequência de consumo } \\
\text { de frituras, salgadinhos e } \\
\text { embutidos }\end{array}$ & $37(65)$ & $20(35)$ & $33(72)$ & $13(28)$ & $04(36)$ & $7(64)^{*}$ \\
\hline $\begin{array}{l}\text { Q11. Frequência de consumo } \\
\text { de doces, refrigerantes e sucos } \\
\text { industrializados }\end{array}$ & $34(60)$ & $23(40)$ & $29(63)$ & $17(37)$ & $05(45)$ & $06(55)$ \\
\hline $\begin{array}{l}\text { Q12. Tipo de gordura usado } \\
\text { para cozinhar }\end{array}$ & $55(97)$ & $02(3)$ & $44(96)$ & $02(4)$ & $11(100)$ & - \\
\hline $\begin{array}{l}\text { Q13. Acréscimo de sal em } \\
\text { refeições já prontas }\end{array}$ & $54(95)$ & $03(5)$ & $45(98)$ & $01(2)$ & $09(82)$ & $02(18)^{*}$ \\
\hline $\begin{array}{l}\text { Q14. Quantidade de refeições } \\
\text { diárias }\end{array}$ & $16(28)$ & $14(72)$ & $15(33)$ & $31(67)$ & $01(9)$ & $10(91)$ \\
\hline Q15. Consumo diário de água & $20(35)$ & $37(65)$ & $17(37)$ & $29(63)$ & $03(27)$ & $08(73)$ \\
\hline $\begin{array}{l}\text { Q16. Frequência de consumo } \\
\text { de bebidas alcoólicas }\end{array}$ & $39(68)$ & $18(32)$ & $31(67)$ & $15(33)$ & $08(73)$ & $03(27)$ \\
\hline $\begin{array}{l}\text { Q17. Frequência semanal de } \\
\text { atividade física }\end{array}$ & $02(3)$ & $55(97)$ & $02(4)$ & $44(96)$ & - & $11(100)$ \\
\hline $\begin{array}{l}\text { Q18. Leitura de informações } \\
\text { nutricionais em rótulos }\end{array}$ & $08(14)$ & $49(86)$ & $07(15)$ & $39(85)$ & $01(9)$ & $10(91)$ \\
\hline
\end{tabular}

de vida, a maioria dos frequentadores apresentou classificação boa + muito boa $(64 \%)$ (Tabela 3).

Também foram calculados os coeficientes de correlação de Pearson entre as variáveis antropométricas, sociodemográficas e a qualidade de vida geral e em seus domí- nios. Observou-se correlação positiva entre o domínio psicológico e a idade $(r=0,292$ e $p=0,028)$ e negativa com o IMC ( $r=-0,308$ e $p=0,02)$. Houve correlação positiva entre o domínio das relações sociais e a idade $(r=0,293$ e $p=0,27$ ), embora sejam correlações fracas (Dados não demonstrados). 
Tabela 3. Qualidade de vida de frequentadores de uma policlínica universitária da zona sul de São Paulo, 2018.

\begin{tabular}{|c|c|c|c|c|c|c|c|c|c|}
\hline \multirow{3}{*}{$\begin{array}{c}\text { Domínios } \\
\text { geral }\end{array}$} & \multirow[b]{2}{*}{ Todos } & \multicolumn{2}{|c|}{$\begin{array}{l}\text { Muito baixa } \\
\text { + baixa }\end{array}$} & & \multicolumn{2}{|c|}{ Regular } & \multirow[b]{2}{*}{ Todos } & \multicolumn{2}{|c|}{ Boa + muito boa } \\
\hline & & $\mathrm{M}$ & $\mathrm{H}$ & Todos & $M$ & $\mathrm{H}$ & & $\mathrm{M}$ & $\mathrm{H}$ \\
\hline & \multicolumn{9}{|c|}{$(\%)$} \\
\hline Físico & $13(23)$ & $10(22)$ & $03(27)$ & $15(26)$ & $13(28)$ & $02(18)$ & $29(51)$ & $23(50)$ & $06(55)$ \\
\hline Psicológico & $03(5)$ & $02(4)$ & $01(9)$ & $14(25)$ & $13(28)$ & $01(9)$ & $40(70)$ & $31(67)$ & $09(82)$ \\
\hline \multirow{2}{*}{$\begin{array}{l}\text { Relações } \\
\text { Sociais } \\
\text { Ambiente }\end{array}$} & $03(5)$ & $01(2)$ & $02(18)$ & $15(26)$ & $14(30)$ & $01(9)$ & $39(68)$ & $31(67)$ & $08(73)$ \\
\hline & $02(4)$ & $02(4)$ & - & $28(49)$ & $21(46)$ & $07(64)$ & $27(47)$ & $23(50)$ & $04(36)$ \\
\hline \multicolumn{10}{|c|}{ Classificação Geral } \\
\hline & \multicolumn{3}{|c|}{ Muito baixa + Baixa } & \multicolumn{3}{|c|}{ Regular } & \multicolumn{3}{|c|}{ Boa + Muito boa } \\
\hline & \multicolumn{9}{|c|}{$(\%)$} \\
\hline Mulher & \multirow{3}{*}{\multicolumn{3}{|c|}{$\begin{array}{l}01(2) \\
01(9) \\
02(4)\end{array}$}} & \multirow{3}{*}{\multicolumn{3}{|c|}{$\begin{array}{l}14(31) \\
04(36) \\
18(32)\end{array}$}} & \multirow{3}{*}{\multicolumn{3}{|c|}{$\begin{array}{l}31(67) \\
06(55) \\
37(64)\end{array}$}} \\
\hline Homem & & & & & & & & & \\
\hline Total Geral & & & & & & & & & \\
\hline
\end{tabular}

M: Mulheres; H: Homens.

\section{DISCUSSÃO}

Os dados do presente estudo mostram que o perfil dos frequentadores desta policlínica universitária são mulheres, adultas, de classe socioeconômica $\mathrm{C}$, com oito anos ou menos de escolaridade, alta prevalência de obesidade, consumo alimentar e outros aspectos do estilo de vida inadequados e qualidade de vida geral boa e muito boa.

O Vigitel Brasil 2018 verificou, na população adulta brasileira, a frequência de $19,8 \%$ de obesidade, com prevalências maiores em mulheres $(20,7 \%)$ do que em homens $(18,7 \%)^{1}$. Neste estudo a prevalência também foi maior nas mulheres (74\%) do que nos homens (64\%), mas no total da amostra estudada é muito superior aos dados nacionais (72\%), em uma amostra composta exclusivamente por participantes com excesso de peso.

$\mathrm{O}$ aumento da escolaridade impacta positivamente na redução da prevalência de obesidade no Brasil ${ }^{1}$ e uma das características da maioria da amostra estudada foram a baixa escolaridade e classe socioeconômica. Um estudo de base populacional com 758 mulheres com mais de 20 anos em Duque de Caxias - RJ, verificou 23\% de obesidade de acordo com o IMC e relação inversa e significante como anos de escolaridade. ${ }^{18}$

Os dados do Vigitel Brasil 2018 demonstraram que no Brasil, o consumo regular de frutas e hortaliças foi de $33,9 \%$ sendo menor entre os homens $(27,7 \%)$ do que em mulheres $(39,2 \%)^{19}$. Neste estudo verificou-se consumo adequado de frutas, verduras e legumes de $26 \%$ e $17 \%$, respectivamente, sendo inferiores aos do Brasil.

Em revisão não sistemática realizada por Machado, Feferbaum e Leone ${ }^{20}$, verificou-se que o consumo de frutas, verduras e legumes é insuficiente em todas as regiões do 
Brasil e em todas as faixas etárias, sendo que o consumo é mais adequado entre as mulheres, com maior renda e nível socioeconômico, o que poderia explicar os baixos dados de consumo adequado desses alimentos. Sabe-se que o consumo adequado de frutas, verduras, legumes e leguminosas se relaciona com o melhor controle do peso corporal. ${ }^{20}$

A inclusão de produtos lácteos em dietas com restrição de energia afeta significativamente a redução do peso, massa de gordura corporal e circunferência da cintura em comparação com as dietas usuais de perda de peso. ${ }^{21}$ No presente estudo os participantes apresentavam excesso de peso e $93 \%$ deles tinham consumo inadequado, abaixo do recomendado para leite e derivados. Isso indica a necessidade de reforço de medidas que valorizem e incentivem essa prática alimentar em todos os grupos etários, frente a alta prevalência de excesso de peso da população brasileira.

Percebe-se que a adição de sal em refeições já prontas está abaixo dos achados nacionais, totalizando $5 \%$ neste estudo, onde os homens adicionavam mais sal aos alimentos que as mulheres (18\% versus $2 \%, p$ $=0,03)$. Nesse sentido, valores inferiores de acréscimo de sal às refeições prontas quando comparados aos dados nacionais, podem-se relacionar com a maior prevalência de mulheres na amostra estudada. Esse hábito é mais frequente em homens que em mulheres, o que foi demonstrado pela diferença significativa na comparação entre os sexos.

O Vigitel 2013, na avaliação da percepção do entrevistado sobre o seu consumo de sal, verificou consumo considerado alto ou muito alto em $16 \%$ da população, com consu- mo maior, também entre os homens $(17,9 \%$ versus $14,3 \%)^{22}$.

Westenhoefer ${ }^{23}$, verificou que muIheres se preocupam mais com as escolhas alimentares que os homens, na busca pelo controle de peso, crenças de saúde e maior conhecimento nutricional dos alimentos, o que pode explicar a maior prevalência de alguns comportamentos saudáveis quando comparadas aos homens.

Outros hábitos saudáveis, como prática de atividade física e consumo frequente de peixes também estão inadequados nessa população ( $97 \%$ e $88 \%$ respectivamente), acompanhando os dados nacionais, que indicam que a população apresenta baixo índice de prática de atividade física e consumo de pescados. ${ }^{1}$

A alimentação e nutrição adequadas são necessárias para a promoção e proteção da saúde para o desenvolvimento pleno do indivíduo, exercendo o direito humano à alimentação adequada e com qualidade de vida. ${ }^{24}$ Além disso, classificam-se como condições mínimas e universais para a qualidade de vida, uma vez que, diz respeito às necessidades mais elementares da vida humana junto ao saneamento básico, condições de moradia, trabalho, saúde, educação e lazer. ${ }^{25}$

Neste estudo foi observada relação negativa e significante entre o domínio psicológico da qualidade de vida e o IMC. Este domínio leva em consideração os aspectos relacionados ao sentido da vida, à dimensão emocional, autoestima, imagem corporal, capacidade de concentração, satisfação consigo próprio, presença de sentimentos positivos e negativos, e espiritualidade. ${ }^{15}$

Estudo que avaliou 200 mulheres com 
média de idade de 52,8 anos, cadastradas no Ambulatório de Climatério da Fundação da Universidade de Caxias do Sul, observou associação negativa e significante, semelhante aos nossos dados, entre os domínios somatovegetativo e psicológicos e o IMC. Estes domínios do instrumento Menopause Rating Scale (MRS) avaliam aspectos relacionados aos sintomas do climatério, além de tristeza, irritabilidade, agressividade, ansiedade, esgotamento físico e mental. ${ }^{26}$

Também houve associação positiva e significante entre o domínio psicológico e a idade. Talvez com o envelhecimento, o senso de estética corporal seja alterado para questões relacionadas à saúde. Verifica-se melhor aceitação das mudanças corporais inerentes ao envelhecimento, priorizando a funcionalidade em relação aos atributos físicos tão valorizados pelos jovens. ${ }^{27}$

O domínio das relações sociais da qualidade de vida se relacionou positivamente com a idade. Kalkman, Koetz e Adami ${ }^{28}$ estudaram 54 mulheres socialmente ativas e observaram a mesma associação. No nosso estudo, $67 \%$ das mulheres apresentaram percepção boa e muito boa neste domínio, e no estudo citado, esta percepção foi de $92,6 \%$, onde os autores sugerem que esta associação pode ser determinada pela participação das mesmas em grupos de convivência e pelos laços de amizade formados ali. O grupo estudado frequenta a policlínica universitária em vários dias da semana, o que facilita o convívio com outros frequentadores, profissionais e colaboradores que permite ampliar as relações sociais.
A menor pontuação de qualidade de vida foi observada no domínio ambiente. Segundo o Índice Paulista de Vulnerabilidade Social (IPVS), da Fundação Sistema Estadual de Análise de Dados (SEADE/SP) ${ }^{29}$, o entorno da policlínica, local de moradia dos frequentadores, situa-se em área classificada em vulnerabilidade social. As populações vulneráveis, principalmente nos centros urbanos, têm dificuldades para acumular capital social, seja individual, coletivo ou cívico, e esta dificuldade é expressa em níveis de qualidade de vida inferiores. ${ }^{30}$

Os achados deste estudo confirmam os dados de pesquisas atuais e reforçam a importância de ações de promoção da saúde que envolvam a população em todas as fases do processo educativo para o gerenciamento de sua própria saúde de acordo com suas necessidades e a realidade local.

Esse estudo apresentou algumas limitações como amostra por conveniência, maior prevalência do sexo feminino e a não avaliação da atividade física.

\section{CONCLUSÃO}

Conclui-se que o grupo estudado foi composto, em sua maioria, por mulheres, obesas,sem companheiro, com baixa escolaridade e classe socioeconômica. Os aspectos do consumo alimentar e outros hábitos de vida apontam para a necessidade de mudanças em busca de comportamentos mais saudáveis, embora a qualidade de vida geral tenha sido relatada pela maioria como boa e muito boa. 


\section{REFERÊNCIAS}

1. Agência Nacional de Saúde Suplementar. Vigitel Brasil 2018: vigilância de fatores de risco e proteção para doenças crônicas por inquérito telefônico : estimativas sobre frequência e distribuição sociodemográfica de fatores de risco e proteção para doenças crônicas nas capitais dos 26 estados brasileiros e no Distrito Federal em 2018. Brasília; 2019.

2. Melendez G., Pimenta AM., Kac G. Epidemiologia do sobrepeso e da obesidade e seus fatores determinantes em Belo Horizonte (MG), Brasil: estudo transversal de base populacional. Rev Panam Salud Públic. 2004; 16(5):308-314.

3. Latinoamericana de Sociedades de Obesidad (Flaso). II Consenso Latino americano de Obesidad, 2017.

4. Cristóvão MF., Sato APS., Fujimori E. Excesso de peso e obesidade abdominal em mulheres atendidas em Unidade de Estratégia de Saúde da Família. Rev Esc Enferm USP. 2011 45(2):1667-1672

5. World Health Organization. Obesity: preventing and managing the global epidemic: report of a WHO consultation on obesity. Geneva, Switzerland; 1997.

6. Associação Brasileira para o Estudo da Obesidade e da Síndrome Metabólica (ABESO). Diretrizes Brasileiras da Obesidade. São Paulo; 2016.

7. Brasil. Ministério da Saúde. Secretaria-Executiva. Departamento de Monitoramento e Avaliação do SUS. Política Nacional de Informação e Informática em Saúde. Brasília; 2016.

8. Frisancho, AR. Anthropometric standards of assessment of growth and nutritional status. Ann Arbor: University of Michigan, 1999.

9. Brasil. Ministério da Saúde. Secretaria de Atenção à Saúde. Departamento de Atenção Básica. Orientações para a coleta e análise de dados antropométricos em serviços de saúde : Norma Técnica do Sistema de Vigilância Alimentar e Nutricional - SISVAN. Brasília; 2011.

10. World Health Organization. Obesity: preventing and managing the global epidemic: report of a WHO consultation on obesity. Geneva, Switzerland; 2000. (WHO Technical Report Series; n. 894).

11. Health Organization. Physical status: the use and interpretation of anthropometry. Geneva, Switzerland: WHO; 1995. (WHO Technical Report Serie; n. 854).

12. Organização Pan-Americana da Saúde. XXXVI Reunión del Comitê Asesor de Ivestigaciones en Salud - Encuestra Multicêntrica - Salud Beinestar y Envejecimeiento (SABE) en América Latina e el Caribe - Informe Preliminar. 2002
13. Associação Brasileira de Empresas de Pesquisa. Critério de classificação econômica Brasil [Internet]. São Paulo; 2012.

14. Brasil. Ministério da Saúde. Guia alimentar: como ter uma alimentação saudável. Brasília: Ministério da Saúde. 2017

15. Fleck MPA., Louzada S., Xavier M., Chachamovich E., Vieira E., Santos L., Pinzon V. Aplicação da versão em português do instrumento abreviado da qualidade de vida "WHOQOL-bref". Rev. Saúde Pública.2000.

16. Batterham AM., Hopkins WG. Making meaningful inferences about magnitudes. Int J Sports Physiol Perform. 2006. 1(1):50-57.

17. Mukaka M. Statistics corner: A guide to appropriate use of correlation coefficient in medical research. Malawi Med J. 2012; 24(3):69-71.

18. Lins APM., Sichieri R., CoutinhoWF., Ramos EG., Peixoto MVM., Fonseca VM. Alimentação saudável, escolaridade e excesso de peso entre mulheres de baixa renda. Ciênc. Saúde Coletiva. 2013; 18(2):357-366.

19. Brasil. Ministério da Saúde. Secretaria de Vigilância em Saúde. Departamento de Vigilância de Doenças e Agravos não Transmissíveis e Promoção da Saúde. Vigitel Brasil 2014: vigilância de fatores de risco e proteção para doenças crônicas por inquérito telefônico: estimativas sobre frequência e distribuição sociodemográfica de fatores de risco e proteção para doenças crônicas nas capitais dos 26 estados brasileiros e no Distrito Federal em 2014. Brasília; 2015. [

20. Machado RHV., Feferbaum R., Leone C. Consumo de frutas no Brasil e prevalência de obesidade. J. Hum. Growth Dev. 2016; 26(2):243-252.

21. Abargouei AS., Janghorbani M., Salehi-Marzijarani M., Esmaillzadeh A. Effect of dairy consumption on weight and body composition in adults: a systematic review and meta-analysis of randomized controlled clinical trials. Int J Obes (Lond). 2012; 36(12): 1485-1493.

22. Agência Nacional de Saúde Suplementar. Vigitel Brasil 2013: vigilância de fatores de risco e proteção para doenças crônicas por inquérito telefônico: estimativas sobre frequência e distribuição sociodemográfica de fatores de risco e proteção para doenças crônicas nas capitais dos 26 estados brasileiros e no Distrito Federal em 2013 Brasília; 2014.

23. Westenhoefer J. Age and gender dependent profile of food choice. Forum Nutr. 2005.

24. Brasil. Ministério da Saúde. Secretaria de Atenção à Saúde. Departamento de Atenção Básica. Política Nacional de Alimentação e Nutrição. Brasília; 2013. 
25. Minayo MCS., Hartz ZMA., Buss PM. Qualidade de vida e saúde: um debate necessário.Ciênc. Saúde coletiva. 2000; 5(1):07-18.

26. Gallon CW., Wender MCO. Estado nutricional e qualidade de vida da mulher climatérica. Rev Bras Ginecol Obstet. 2012; 34(4):175-183.

27. Menezes TN., Brito KQD., Oliveira ECT., Pedraza DF. Percepção da imagem corporal e fatores associados em idosos residentes em município do nordeste brasileiro: um estudo populacional. Ciênc. Saúde Coletiva. 2014.

28. Kalkmann ICM., Koetz LCE., Adami FS. Estado nutricional e a qualidade de vida de mulheres. Cad. Pedagógico. 2015; 12(1):161-173.
29. Ferreira MP. Índice Paulista de Vulnerabilidade Social. São Paulo: Fundação SEADE;2013.

30. Silva AV. Vulnerabilidade social e suas consequências:o contexto educacional da juventude na região metropolitana de natal. $13^{\circ}$ Encontro de Ciências Sociais do Norte Nordeste; Maceió: Universidade Federal de Alagoas; 2007.

\section{CORRESPONDÊNCIA}

Márcia Salgueiro

marciasalgueironutricionista@yahoo.com.br 